\title{
ADAPTIVE TRAJECTORY TRACKING CONTROL OF A QUADROTOR BASED ON ITERATIVE LEARNING ALGORITHM
}

\author{
Mohammad Mehdi Farzaneh $^{\mathrm{a}^{*}(\mathbb{D}) \text {, Alireza Tavakolpour-Saleh }}{ }^{\mathrm{a}}$ \\ ${ }^{a}$ Department of Mechanical Engineering, Shiraz University of Technology, Iran \\ m.farzaneh@sutech.ac.ir (" corresponding author),tavakolpour@sutech.ac.ir
}

\begin{abstract}
This paper presents a new adaptive and optimal algorithm for the trajectory tracking control of a quadrotor using iterative learning algorithm (ILA) and enumerative learning method. Ordinarily the ILA, as an adaptive method, can perform well with PID control to improve the controller's performance for a nonlinear system. Quadrotors are considered as nonlinear and unstable systems in which the use of an adaptive and optimal controller can increase its stability and decrease error level. In this method, a PID controller is proposed for the inner and outer control loops of a quadrotor and the ILA is used to adapt PID control gains. Subsequently, an enumerative learning algorithm is used to optimize the learning rates of the ILA. For this purpose, at first, the dynamic model of the quadrotor is acquired. After that, the structure of the control system and the inner and outer control loops are defined. In the end, the simulation results for the trajectory tracking control are demonstrated. Through simulation, it is concluded that as time increases, the performance of the suggested control method in trajectory tracking control becomes better and better and error signals convergence to zero.
\end{abstract}

Keywords: Quadrotor, trajectory tracking control, PID control, iterative learning algorithm

\section{Introduction}

Quadrotor is the four-propeller type of unmanned aerial vehicles and has a wide range of utilities in civilian and military applications. Basically, the quadrotor differs from the common helicopter, which uses rotors with dynamically varying pitch blades. Quadrotor's smaller blades are a useful feature since they contain less kinetic energy thus reducing their ability to cause damage. Owing to the simple and low-cost build and high maneuverability, quadrotors are the most common and popular flying robots in the world. The quadrotor has four inputs and six coupled outputs. Moreover, it is considered as an under-actuated and highly nonlinear system. So, design and implement a control system for quadrotor had been a challenge in recent years. 
PID control algorithm is a common method in control and robotics. A cascade control based on generalized predictive controller and PID controller was applied to regulate the position of a quadrotor [1]. A recursive algorithm for a PID controller was designed to simplify the calculation stages concerning the tracking errors [2]. A PID controller with an extended Kalman filter was studied to filter out the sensors and system noises [3]. Fuzzy control logic was developed to adjust classic PD and PID control for quadrotor stability control [4]. To deal with uncertainties, a linear quadratic regulator controller was applied for better stabilization and improving the flight quality of quadrotor under noisy sensor measurements [5]. A sliding mode controller was obtained based on the back-stepping method for stabilization of a quadrotor [6]. Model reference adaptive and fixed gain linear quadratic regulator controllers were implemented for a quadrotor with parametric uncertainties [7]. An intelligent control algorithm based on particle swarm optimization was used to increase the performance of controllers in the presence of disturbances $[8,9]$.

There are several algorithms for trajectory tracking control of the quadrotor. A Fuzzy control was applied to set the PID-ILC parameters to restrain the influence of uncertain factors and improve the control precision [10]. A combination of Fuzzy control and PID control was applied to tune the control parameters of a model reference adaptive control for the altitude and attitude control of a tricopter [11]. A combination of the integral back-stepping control with the sliding mode control was studied for stabilization of a quadrotor attitude and to accomplish the task of trajectory tracking control $[12,13]$. The effectiveness of a PID control and a back-stepping control for trajectory tracking control was evaluated by simulation results [14]. An adaptive controller was designed for an autonomous quadrotor using nonlinear dynamic inversion and neuro-adaptive methods [15]. A geometric sliding mode controller was designed to improve the robustness and accelerate the convergence rate of trajectory tracking control [16].

In this paper, an adaptive and optimal control algorithm is designed for the trajectory tracking control of quadrotor. Typically, the ILA is considered as an adaptive algorithm through which the performance of a control system becomes better and better as time increases. By this mean, a PID controller adapted by the P-type ILA is used for attitude control and a PID controller adapted by the PID-type ILA is used for altitude and position control. Meanwhile, an enumerative learning algorithm is designed to select the optimum learning rates for the ILA. Therefore, the layout of this paper is as follows. In Section 2, the complete 6-DOF dynamic model of the quadrotor is discussed. The structures of the control system and the inner and outer control loops are described in Section 3. Section 4 illustrates the performance of the baseline control method in trajectory tracking control through simulation. Finally, in Section 5 this document is terminated with the conclusion and the inspiration that can be derived from this work.

\section{Quadrotor modeling}

The complete 6-DOF dynamic model of the quadrotor is presented in this section. For this mean, the system of coordinates to use should be defined first. There are two reference frames: an earth frame indicated by the " $e$ " index and a body-fixed frame indicated by the " $b$ " index. The earth frame is located at the operator's position and the body-fixed frame origin is coinciding with the center of the gravity. Figure 1 shows a conceptual scheme of the quadrotor, reference frames, and Euler angles. 


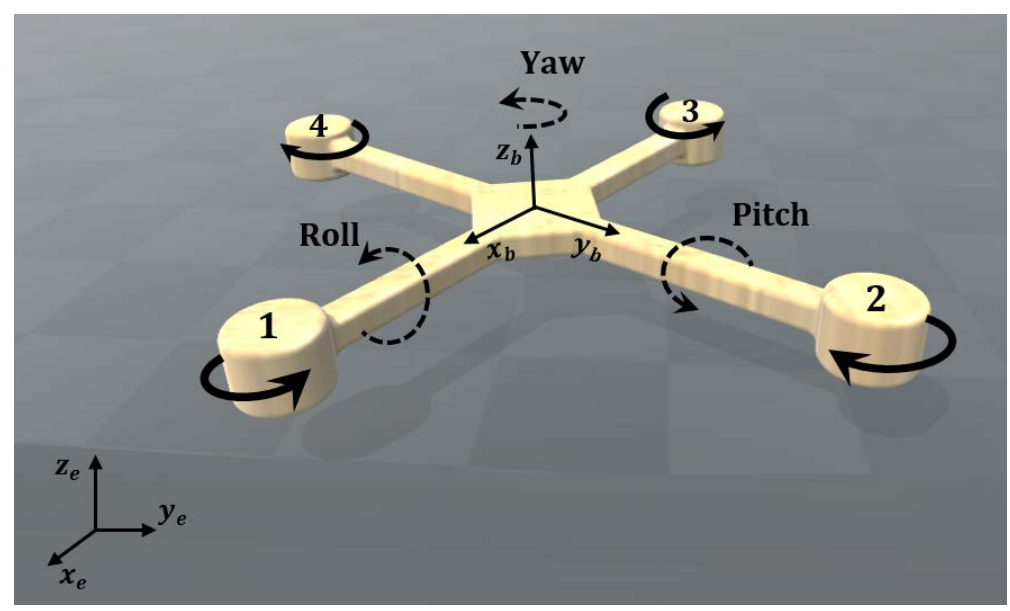

Figure 1. Quadrotor scheme

The quadrotor generally uses two pairs of rotors and to cancel the net moment along the zaxis, one pair (rotors 1 and 3) should rotate clockwise and one pair (rotors 2 and 4) counterclockwise. By changing the rotational speed of each rotor, the quadrotor supplants from a point to another through space. To make the y-axis torque and then the pitch angle $(\theta)$, one can augment the speed of rotor 3 and reduce the speed of rotor 1 . This action causes the quadrotor to move along the $x$-axis. Likewise, to make the $x$-axis torque and then the roll angle $(\varphi)$, one can augment the speed of rotor 2 and reduce the speed of rotor 4 . This action also causes the quadrotor to move along the y-axis. Finally, to increase the z-axis torque and then the yaw angle $(\psi)$, one can augment the speed of rotors 1 and 3 and reduce the speed of rotors 2 and 4.

To drive dynamic equations with respect to the earth frame, a rotation and a translational matrix should be used. The rotation matrix given by (1) is used to state linear velocities with respect to the earth frame. Also, the reverse translational matrix given by (2) is used to convert angular velocities with respect to the body-fixed frame to angular velocities with respect to the earth frame.

$R=\left[\begin{array}{ccc}\cos \theta \cos \psi & \sin \varphi \sin \theta \cos \psi-\cos \varphi \sin \psi & \cos \varphi \sin \theta \cos \psi+\sin \varphi \sin \psi \\ \cos \theta \sin \psi & \cos \varphi \cos \psi+\sin \varphi \sin \theta \sin \psi & \sin \theta \cos \varphi \sin \psi-\sin \varphi \cos \psi \\ -\sin \theta & \sin \varphi \cos \theta & \cos \varphi \cos \theta\end{array}\right]$,

$T=\left[\begin{array}{ccc}1 & \tan \theta \sin \varphi & \tan \theta \cos \varphi \\ 0 & \cos \varphi & -\sin \varphi \\ 0 & \sin \varphi / \cos \theta & \cos \varphi / \cos \theta\end{array}\right]$

The Newton-Euler equations given by (3) and (4) are used to drive the nonlinear dynamic model of the quadrotor.

$$
\begin{aligned}
& F_{n e t}=\frac{d}{d t}[m V]_{b}+\Omega \times[m V]_{b}, \\
& M_{n e t}=\frac{d}{d t}[I \Omega]_{b}+\Omega \times[I \Omega]_{b},
\end{aligned}
$$


The quadrotor is assumed to be symmetric inherent to the $\mathrm{x}$ and $\mathrm{y}$-axis. It means that the center of gravity is located at the center of the quadrotor. Also, angles variations are small and the motor inertia is negligible rather than the quadrotor inertia. By considering these assumptions, the 6-DOF dynamic model with respect to the body-fixed frame can be expressed as Equations (5)-(10) [17-19].

$\ddot{X}=\frac{1}{m} U_{1}(\cos \varphi \sin \theta \cos \psi+\sin \varphi \sin \psi)$,

$\ddot{Y}=\frac{1}{m} U_{1}(\sin \theta \cos \varphi \sin \psi-\sin \varphi \cos \psi)$,

$\ddot{Z}=\frac{1}{m} U_{1}(\cos \varphi \cos \theta)-g$,

$\ddot{\varphi}=\frac{1}{I_{x x}} U_{2}-\left(\frac{I_{z z}-I_{y y}}{I_{x x}}\right) \dot{\theta} \dot{\psi}$,

$\ddot{\theta}=\frac{1}{I_{y y}} U_{3}-\left(\frac{I_{x x}-I_{z z}}{I_{x x}}\right) \dot{\varphi} \dot{\psi}$,

$\ddot{\psi}=\frac{1}{I_{z z}} U_{4}-\left(\frac{I_{y y}-I_{x x}}{I_{z z}}\right) \dot{\varphi} \dot{\theta}$,

where $m$ and $I$ stand for the quadrotor's mass and inertia matrix respectively. Also, $U_{i}$ is manipulated variable so that, $U_{1}$ is related to the thrust force, $U_{2}$ to the y-axis torque, $U_{3}$ to the $\mathrm{x}$-axis torque, and $U_{4}$ to the z-axis torque. Basically, the quadrotor motion is achieved by changing the combination and varying the speed of the rotors described as Equations (11)(14) [20].

$U_{1}=k_{T}\left(w_{1}^{2}+w_{2}^{2}+w_{3}^{2}+w_{4}^{2}\right)$

$U_{2}=k_{T} l\left(w_{3}^{2}-w_{1}^{2}\right)$,

$U_{3}=k_{T} l\left(w_{2}^{2}-w_{4}^{2}\right)$,

$U_{4}=k_{D}\left(w_{1}^{2}-w_{2}^{2}+w_{3}^{2}-w_{4}^{2}\right)$,

where $l, k_{T}, k_{D}$, and $w$ stand for the quadrotor's radius, thrust coefficient, drag factor, and propeller's speed respectively. Parameters $m, I$, and $l$ are physical characteristics and $k_{T}, k_{D}$, and $w$ are measured based on motor's property.

\section{Control scheme}

The quadrotor has four inputs and six coupled outputs and is considered as an under-actuated and highly nonlinear system. So a classical PID controller cannot handle is nonlinear inherent as it is expected. One solution is to use an adaptive algorithm that can adapt PID control in different modes. Here, a combination of ILA and enumerative learning algorithm is applied to select optimum gains for PID control somehow the error signals convergence to their minimum level. 


\subsection{Control system}

Equation (15) is a general form of the control law for a classical PID controller:

$U_{i}=K_{P} \cdot e+\frac{1}{T_{I}} \int e d t+T_{D} \frac{d e}{d t^{\prime}}$

where $K_{P}$ is the proportional gain, $T_{I}$ is the reset time, $T_{D}$ is the rate time, and $e$ is the error signal.

ILA or iterative learning control (ILC) is an adaptive control method through which the performance of a control system becomes better and better as time increases and can improve tracking performance of a nonlinear system. This algorithm can perform well with the various types of controllers. Fuzzy control, neural network control, and genetic algorithm are some other methods that can improve PID control's performance more prices and faster than ILA. Equation (16) shows a general form of the control update law describing a PID-type ILA which includes parameters $e, \int e d t$, and $\frac{d e}{d t}$.

$G_{k+1}^{i}=G_{k}^{i}+\alpha^{i} \cdot e_{k}^{i}+\beta^{i} \int e_{k}^{i} d t+\gamma^{i} \frac{d e_{k}^{i}}{d t}$

where $\alpha, \beta$ and $\gamma$ are the learning rates. For a P-type ILA; gains and are equal to zero. To adapt the PID control gains, parameters $K_{P}, T_{I}$, and $T_{D}$ are set as $G$ each in a separate equation. Figure 2 shows the block diagram of a PID-type ILA.

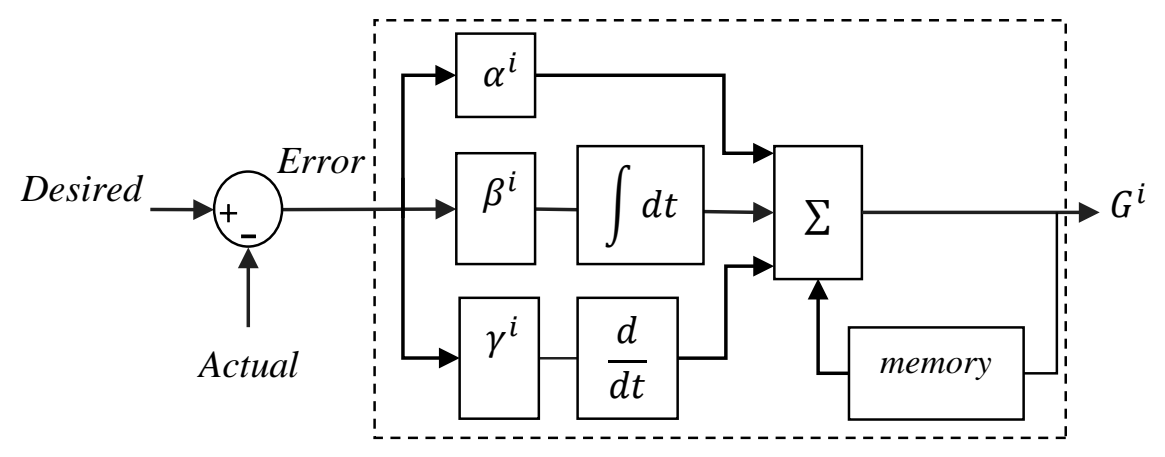

Figure 2. ILA block diagram

To tune the learning rates of ILA, an optimal method is used named as enumerative learning. In this method, a normalized boundary with specific steps selects for each parameter. Then the algorithm runs until the minimum total error is acquired and then the values in that step are saved as the optimal values for the learning rates. Finally, Figure 3 shows the overall block diagram of the proposed control system. 


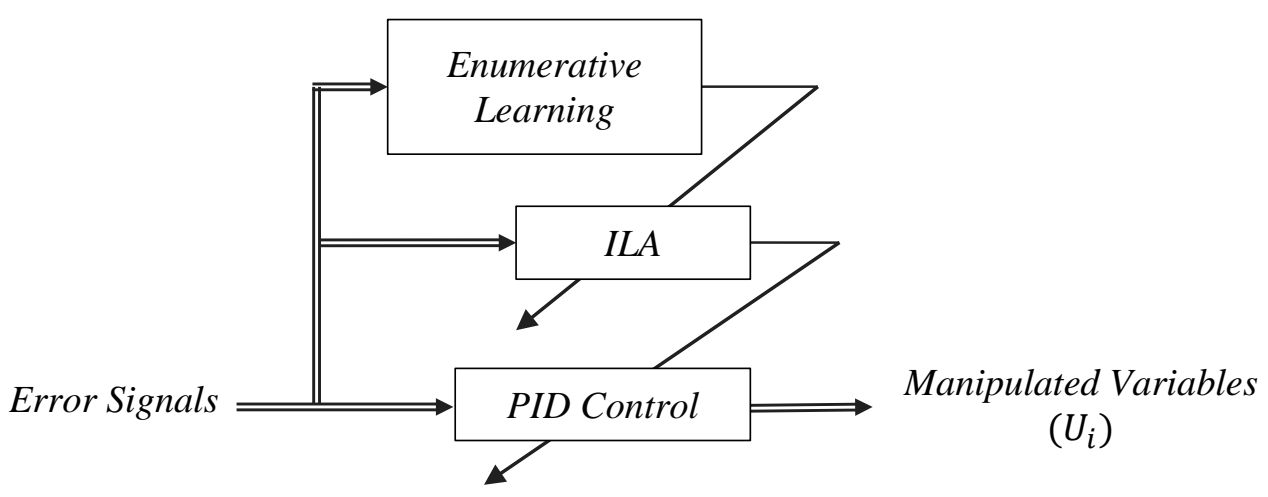

Figure 3. Control system block diagram

\subsection{Control loops}

Altitude and attitude control of the quadrotor is named as the inner control loop. For this part, a PID controller adapted by a PID-type ILA is employed for altitude control while a PID controller adapted by a P-type ILA is used for attitude control. The block diagram of the inner control loop and its input variables (error signals) and output variables (actual states) are shown in Figure 4.

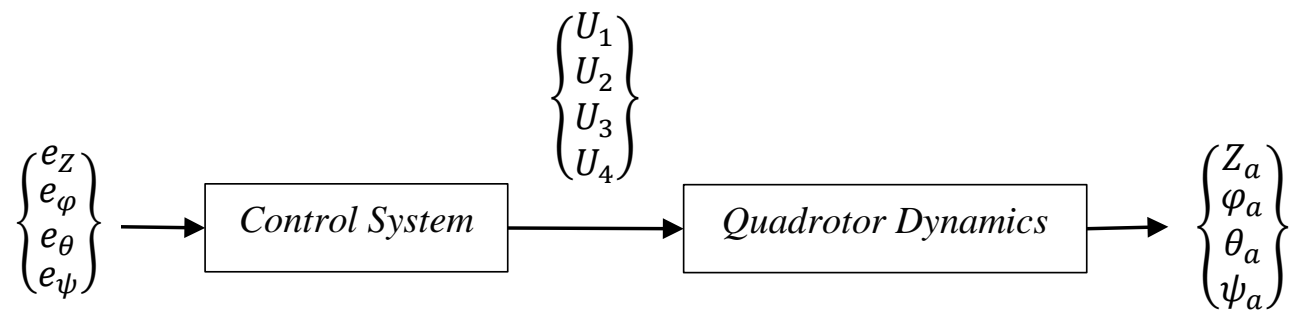

Figure 4. Inner control loop

Position control of the quadrotor is usually named as the outer control loop. For this part, a PID controller adapted by a PID-type ILA is employed for position control. The block diagram of the outer control loop and its input variables (error signals) and output variables (desired angles) are shown in Figure 5.

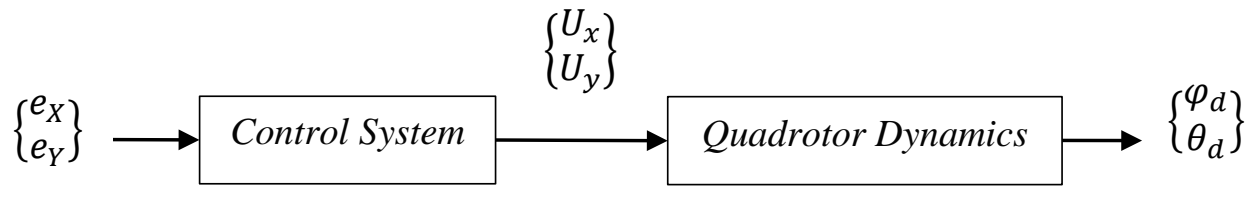

Figure 5. Outer control loop

where the relation between virtual forces $U_{x}$ and $U_{y}$ by the desired angles can be expressed as Equations (17) and (18) [20, 21]. 
$U_{x}=\cos \varphi \sin \theta \cos \psi+\sin \varphi \sin \psi$,

$U_{y}=\sin \theta \cos \varphi \sin \psi-\sin \varphi \cos \psi$,

\section{Discussion simulation results}

In this section, the performance of the proposed control method in trajectory tracking control is illustrated using Simulink MATLAB. The desired trajectories are selected as a circular path, a butterfly path, and a spiral path. Also for the simulation purpose, the imaginary quadrotor parameters are chosen as Table 1 [22].

Table 1. System Parameters for Simulation Purposes.

\begin{tabular}{|c|c|c|c|}
\hline Parameter & Description & Value & Units \\
\hline$m$ & Quadrotor mass & 0.14 & $\mathrm{~kg}$ \\
\hline$l$ & Quadrotor arm & 0.17 & $\mathrm{~m}$ \\
\hline$I_{x x}$ & Inertia moment with respect to the x-axis & 0.002 & $\mathrm{~kg} . \mathrm{m}^{2}$ \\
\hline$I_{y y}$ & Inertia moment with respect to the y-axis & 0.002 & $\mathrm{~kg} \cdot \mathrm{m}^{2}$ \\
\hline$I_{z z}$ & Inertia moment with respect to the z-axis & 0.004 & $\mathrm{~kg} \cdot \mathrm{m}^{2}$ \\
\hline$g$ & Gravity acceleration & 9.8 & $\mathrm{~m} . \mathrm{s}^{-2}$ \\
\hline
\end{tabular}

The simulation result for tracking a circular path and its error signals $\left(X_{e}, Y_{e}, Z_{e}\right)$ are shown in Figure 6. It can be seen that as time increases, the proposed controller is able to move the quadrotor to the desired path and minimize the error signals. 


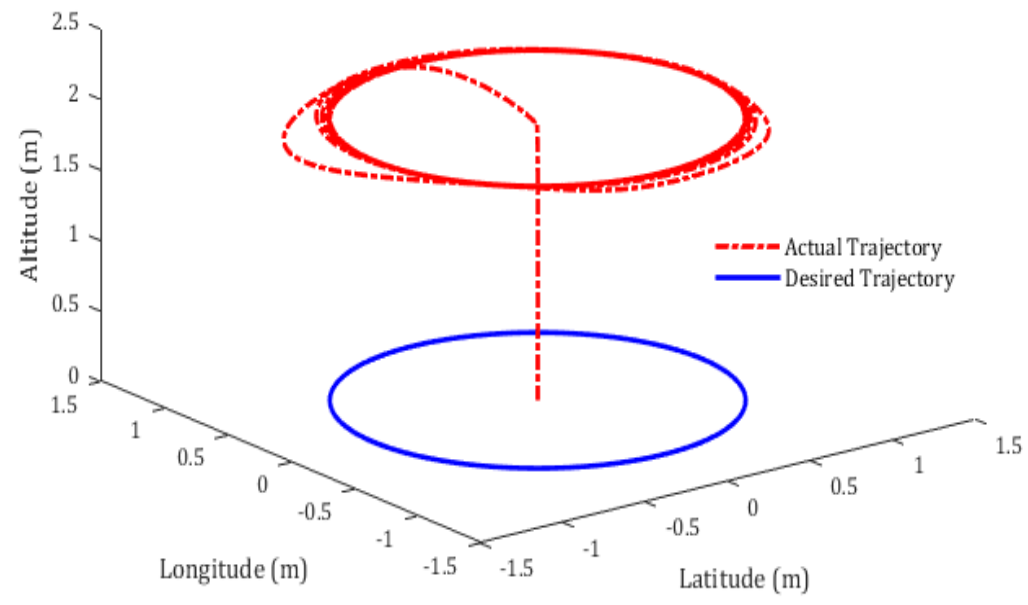

(a)

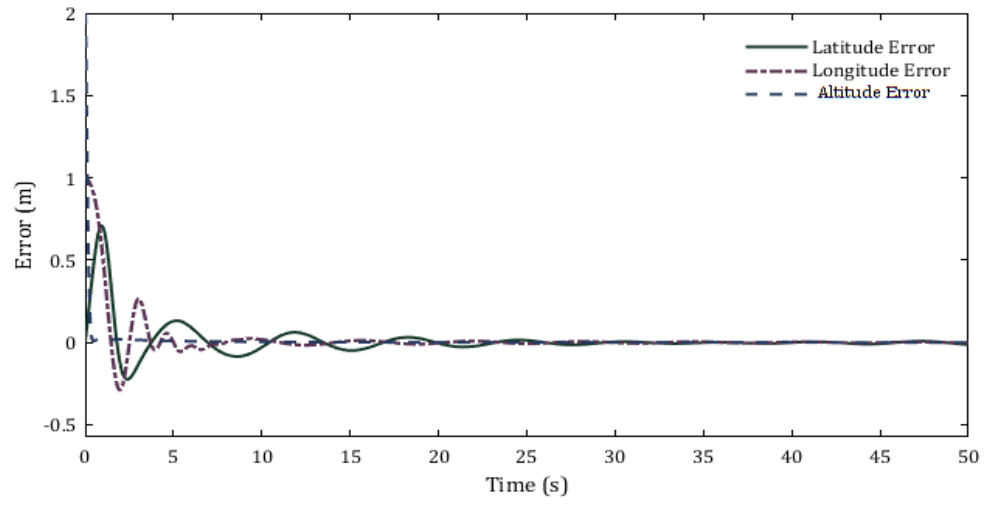

(b)

Figure 6. (a) Trajectory tracking control for a circular path; (b) Error signals

The simulation result for tracking a butterfly path and its error signals $\left(X_{e}, Y_{e}, Z_{e}\right)$ are shown in Figure 7. Just like the circular path, it can be seen that the proposed controller is able to move the quadrotor to the desired path and minimize the error signals as time increases. 


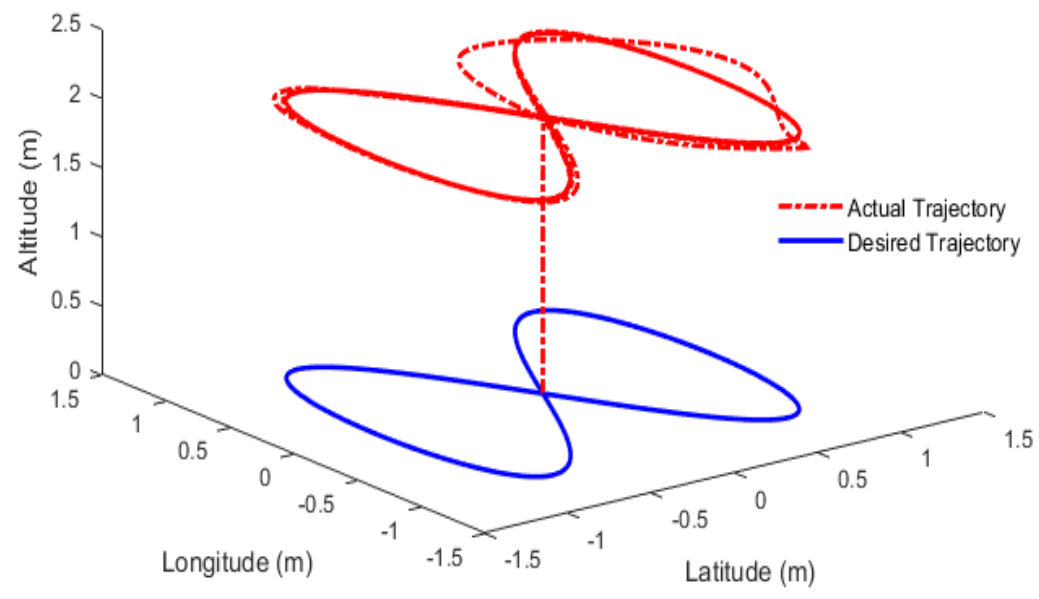

(a)

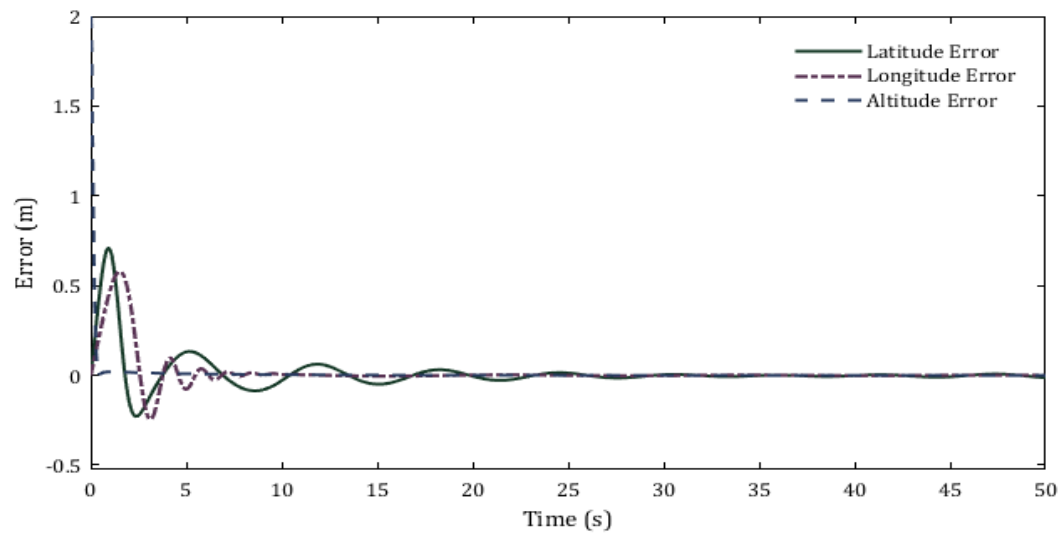

(b)

Figure 7. (a) Trajectory tracking control for a butterfly path; (b) Error signals

The simulation result for tracking a spiral path and its error signals $\left(X_{e}, Y_{e}, Z_{e}\right)$ are shown in Figure 8. Usually, for tracking a trajectory, roll and pitch angles change and this change affects the total thrust which may cause instability. However, the proposed controller is able to move the quadrotor to the desired path and minimize the error signals as time increases. 


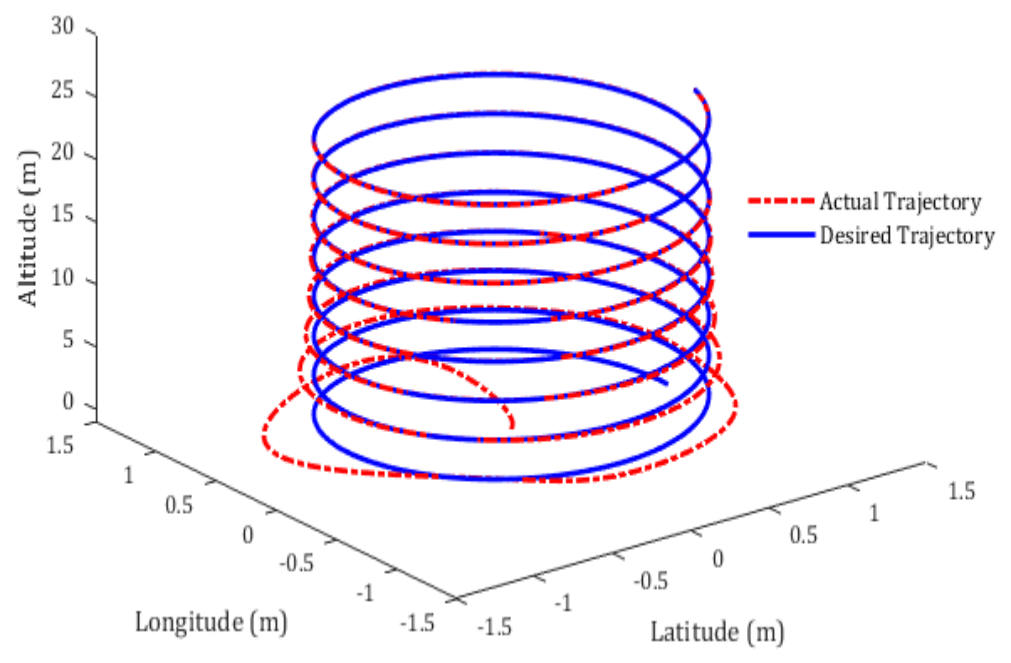

(a)

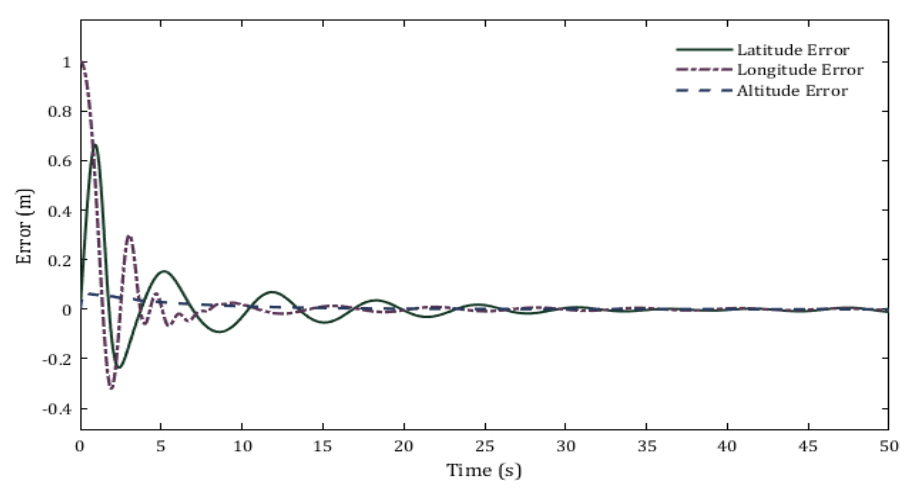

(b)

Figure 8. (a) Trajectory tracking control for a spiral path; (b) Error signals.

\section{Conclusions}

In this paper, an optimal ILA was presented to adapt PID control using the enumerative learning algorithm for the trajectory tracking control of a quadrotor. In this method, a PID controller adapted by the PID-type ILA was proposed for altitude and position control and a PID controller adapted by the P-type ILA was proposed for attitude control of a quadrotor. Also, the enumerative learning algorithm was used to choose the optimal values of the learning rates. For this purpose, at first, the dynamic model of the quadrotor was acquired based on the Newton-Euler equations. After that, the adaptive and optimal control system and the inner and outer control loops were defined. In the end, the simulation results for trajectory tracking of the quadrotor were demonstrated. Through simulation, it is concluded that as time increases, the performance of the suggested control method in trajectory tracking control becomes better and better and error signals convergence to zero. Future work in this area is to implement the proposed control method on an experimental model. 


\section{References}

[1] Criado, R.M, Rubio, F.R., “Autonomous path tracking control design for a commercial quadcopter”, IFAC-PapersOnLine 48(9) (2015) : 73-78.

[2] Salih, A.L., Moghavvemi, M., Mohamed, H.A., Gaeid, K.S., "Flight PID controller design for a UAV quadrotor”, Scientific research and essays 5(23) (2010) : 3660-3667.

[3] Tanveer, M.H., Ahmed, S.F., Hazry, D., Warsi, F.A., Joyo, M.K., “Stabilized controller design for attitude and altitude controlling of quad-rotor under disturbance and noisy conditions”, American Journal of Applied Sciences 10(8) (2013) : 819-831.

[4] Tikani, V., Shahbazi, H., "Design and implementation of attitude PID controller with fuzzy system to adjust the controller gain values for quadrotor”, Modares Mechanical Engineering 16(9) (2016) : 19-28.

[5] Ahmed, S.F., Kadir, K., Joyo, M.K., "LQR-based controller design for altitude and longitudinal movement of quadrotor”, Journal of Applied Sciences 16(12) (2016) : 588593.

[6] Bulgakov, A., Evgenov, A., Weller, C., “Automation of 3D building model generation using quadrotor”, Procedia Engineering 123 (2015) : 101-109.

[7] Ghaffar, A.A, Richardson, T., "Model reference adaptive control and LQR control for quadrotor with parametric uncertainties", International Journal of Mechanical, Aerospace, Industrial, Mechatronic and Manufacturing Engineering 9(2) (2015) : 244250.

[8] Mohammadi, V., Ghasemi, S., Kharrati, H., "PSO tuned FLC for full autopilot control of quadrotor to tackle wind disturbance using bond graph approach”, Applied Soft Computing 65 (2018) : 184-195.

[9] Tran, H.K., Chiou, J.S., "PSO-based algorithm applied to quadcopter micro air vehicle controller design”, Micromachines 7(9) (2016) : 168.

[10] Dong, J., He, B., "Novel fuzzy PID-type iterative learning control for quadrotor UAV”, Sensors 19(1) (2019) : 24.

[11] Ali, Z.A., Wang, D., Aamir, M., Masroor, S., "Trajectory tracking of a tri-rotor aerial vehicle using an MRAC-based robust hybrid control algorithm”, Aerospace 4(1) (2017) : 3-19.

[12] Jia, Z., Yu, J., Mei, Y., Chen, Y., Shen, Y., Ai, X., “Integral backstepping sliding mode control for quadrotor helicopter under external uncertain disturbances”, Aerospace Science and Technology 68 (2017) : 299-307.

[13] Modirrousta, A., Khodabandeh, M., "A novel nonlinear hybrid controller design for an uncertain quadrotor with disturbances”, Aerospace Science and Technology 45 (2015) : 294-308.

[14] Vempati, A.S., Choudhary, V., Behera, L., "Quadrotor: design, control and vision based localization”, IFAC Proceedings Volumes 47(1) (2014) : 1104-1110.

[15] Shao, X., Meng, Q., Liu, J., Wang, H., "RISE and disturbance compensation based trajectory tracking control for a quadrotor UAV without velocity measurements", Aerospace Science and Technology 74 (2018) : 145-159.

[16] Xia, D., Cheng, L., Yao, Y., “A robust inner and outer loop control method for trajectory tracking of a quadrotor”, Sensors 17(9) (2017) : 2147. 
[17] Shastry, A.K., Pattanaik, A., Kothari, M., "Neuro-adaptive augmented dynamic inversion controller for quadrotors”, IFAC-PapersOnLine 49(1) (2016) : 302-307.

[18] Govea-Vargas, A., Castro-Linares, R., Duarte-Mermoud, M.A., Aguila-Camacho, N., Ceballos-Benavides, G.E., "Fractional order Sosliding mode control of a class of second order perturbed nonlinear systems: Application to the trajectory tracking of a quadrotor”, Algorithms 11(11) (2018) : 168.

[19] Emran, B.J., Al-Omari, M., Abdel-Hafez, M.F., Jaradat, M.A., “A cascaded approach for quadrotor’s attitude estimation”, Procedia Technology 15 (2014) : 268-277.

[20] Bouadi, H., Tadjine, M., "Nonlinear observer design and sliding mode control of four rotors helicopter", International Journal of Mechanical, Aerospace, Industrial, Mechatronic and Manufacturing Engineering 1(7) (2007) : 354-359.

[21] Stevek, J., Fikar, M., “Teaching aids for laboratory experiments with AR. Drone2 Quadrotor”, IFAC-PapersOnLine 49(6) (2016) : 236-241.

[22] Gonzalez, V.S., Moreno, V.J., "Motion control of a quadrotor aircraft via singular perturbations”, International Journal of Advanced Robotic Systems 10(10) (2013) : 368. 